\title{
Ischemic postconditioning regulates cardiomyocyte autophagic activity following ischemia/reperfusion injury
}

\author{
LIANG GUO $^{1}$, JUN-MEI XU ${ }^{1}$ and XIAO-YE $\mathrm{MO}^{2}$ \\ ${ }^{1}$ Department of Anesthesiology, The Second Xiangya Hospital; ${ }^{2}$ Department of Emergency, Xiangya Hospital, \\ Central South University, Changsha, Hunan 410011, P.R. China
}

Received May 23, 2014; Accepted February 13, 2015

DOI: $10.3892 / \mathrm{mmr} .2015 .3533$

\begin{abstract}
Ischemic postconditioning (IPostC) is a promising protective mechanism for combating reperfusion injury. However, the role of autophagy in the protective effects of IPostC and the associated signaling pathways have remained to be elucidated. Male Sprague Dawley rats were subjected to $30 \mathrm{~min}$ ischemia and 1, 2, 3, 6, 12 and $24 \mathrm{~h}$ of reperfusion, with or without IPostC treatment. Autophagic flux was evaluated by detecting mRNA and protein expression levels of microtubule associated protein 1 light chain 3 and p62. Phosphorylated (p)-P70S6 kinase (P70S6K), p-adenosine monophosphate-activated kinase (AMPK) and Beclin 1 protein levels were measured by western blot analysis. Myocardial infarct size was measured using staining with Evans blue dye and myocardial apoptosis was evaluated by terminal deoxynucleotidyl transferase mediated dUTP nick end labeling staining. Autophagic activity was observed to be inhibited within the first hour of reperfusion, increased during 2-6 $\mathrm{h}$ and reduced from 12-24 $\mathrm{h}$ following IPostC compared with reperfusion without IPostC. Inhibition of autophagy by chloroquine significantly reversed the effects of IPostC on myocardial infarct size and the levels of apoptosis. IPostC significantly increased Beclin 1 levels, inhibited AMPK activation and increased P70S6K activation within the first hour compared with reperfusion without IPostC. IPostC attenuated the upregulation of Beclin 1 levels, increased AMPK activation and reduced P70S6K activation between 2 and $12 \mathrm{~h}$, and subsequently exerted the opposite effects on these molecules between 12 and $24 \mathrm{~h}$. IPostC was demonstrated to regulate autophagic activity in a time-dependent manner. The Beclin 1 and AMPK-mammalian target of rapamycin signaling pathways are suggested to be involved in the regulation of IPostC in autophagy.
\end{abstract}

Correspondence to: Dr Jun-Mei Xu, Department of Anesthesiology, The Second Xiangya Hospital, Central South University, 86 Renmin Road, Changsha, Hunan 410011, P.R. China E-mail: 2330guoliang@163.com

Key words: autophagy, adenosine monophosphate-activated kinase, Beclin 1, mammalian target of rapamycin, reperfusion injury

\section{Introduction}

Myocardial ischemic postconditioning (IPostC) treatment, applied at the onset of reperfusion, has been reported to effectively ameliorate ischemia/reperfusion (I/R) injury of the heart (1). Previous studies have suggested that the protective effects of IPostC are predominantly established via activation of the reperfusion injury salvage kinase (RISK) pathway and the Janus kinase-signal transducer and activator of transcription pathway by inhibiting mitochondrial permeability transition pore opening (2,3). Previous studies have also indicated that autophagy is involved in the protective mechanisms of IPostC $(4,5)$.

Autophagy is an intracellular degradation pathway, in which long-lived or aggregated proteins and damaged organelles are transported to lysosomes for destruction (6). In the heart, autophagy serves a homeostatic role under physiological conditions, but abnormal autophagy may result in cardiac dysfunction (7). A previous study demonstrated that autophagy was upregulated during myocardial I/R and suggested that it may be cardioprotective during ischemia (8). During myocardial ischemia and reperfusion, autophagy is upregulated through activation of an adenosine monophosphate-activated kinase (AMPK)- and Beclin 1-dependent mechanism (8). The AMPK-mammalian target of rapamycin (mTOR) pathway and Beclin 1 are core autophagy regulators, of which mTOR is additionally involved in the RISK pathway. However, the involvement of autophagy during the reperfusion period remains controversial (8-11). The current study aimed to investigate the role of autophagy in IPostC by comparing the myocardium autophagic flux and alterations in the key autophagy regulators within a $24 \mathrm{~h}$ interval between IPostC and I/R.

\section{Materials and methods}

Animals. A total of 192 male Sprague Dawley rats (SLAC Laboratory Animals Ltd., Hunan, China) weighing 200-300 g were used in the current study. The rats were housed in cages with ad libitum access to food pellets and water, were maintained at $22^{\circ} \mathrm{C}$, and were exposed to a $12 \mathrm{~h}$ light/dark cycle. The animal protocol was approved by the Animal Care and Use Committee of Xiangya Medical College, Central South University (Changsha, China). 
$I / R$ modeling. Rats were anesthetized by intraperitoneal injection of pentobarbital $(60 \mathrm{mg} / \mathrm{kg}$; Sigma-Aldrich, St. Louis, MO, USA) and were placed on a heating pad (XR-YLS-20A; Xinruan Information Technology, Co., Ltd., Shanghai, China) maintained at $36-38^{\circ} \mathrm{C}$. An electrocardiogram was recorded using a Power-Lab monitoring system (BL-410 system; Chengdu Taimeng Science And Technology Co., Ltd., Chengdu, China). A left thoracotomy was subsequently performed to expose the heart at the fourth intercostal space. Ischemia was established by placing a snare on the top of the anterior descending branch of the left coronary artery (LAD) using a 6-0 nylon suture (Cheng-He Microsurgical Instruments Factory, Ningbo, China). Regional ischemia was confirmed by alterations in ST-T on the electrocardiogram.

Western blot assay of autophagy. Rats were randomly divided into I/R, IPostC and Sham-operation groups. Rats in the I/R group were subjected to $30 \mathrm{~min}$ ischemia to the heart followed by $1,2,3,6,12$ and 24 h of reperfusion ( $n=6$ per time-point). Rats in the IPostC group were subjected to $30 \mathrm{~min}$ ischemia to the heart prior to three cycles of 30 -sec reperfusion/30-sec ischemia followed by $1,2,3,6,12$ or $24 \mathrm{~h}$ reperfusion $(n=6$ per time-point). Rats in the Sham-operation group $(n=6)$ underwent the same procedure without occlusion of the vessel. Rats were sacrificed by overdose of anesthesia (pentobarbital, $180 \mathrm{mg} / \mathrm{kg}$ ).

Hearts were excised and left ventricular (LV) muscles were lysed in ice-cold radioimmunoprecipitation assay buffer containing protease and phosphatase inhibitor cocktails (EMD Millipore, Billerica, MA, USA). The concentration of the lysates was measured using a Bicinchoninic Acid Protein Assay kit (Beyotime Institute of Biotechnology, Beijing, China). Total protein $(30 \mu \mathrm{g})$ was separated on $8-15 \%$ SDS polyacrylamide gels (Beyotime Institute of Biotechnology) and transferred onto polyvinylidene difluoride membranes (EMD Millipore). The membranes were blocked with blocking buffer [5\% bovine serum albumin (Wuhan Boster Biological Technology, Ltd., Wuhan, China)-buffered saline containing $0.05 \%$ Tween 20 (Sigma-Aldrich)] for $1 \mathrm{~h}$ at room temperature. The blocked membranes were incubated with primary antibodies overnight at $4^{\circ} \mathrm{C}$. Subsequent to washing (Western Washing Liquid; Beyotime Institute of Biotechnology), the membranes were incubated with horseradish peroxidase-conjugated goat anti-rabbit peroxidase-conjugated secondary antibody (1:1,000; cat. no. A0208; Beyotime Institute of Biotechnology) for $1 \mathrm{~h}$ at room temperature. Protein bands were visualized with the ECL Western Blotting Detection kit (EMD Millipore), and the chemiluminescence signal was detected using X-ray film (Kodak Digital Imaging Products Company Ltd., Xiamen, China) with photographic cassettes (Beyotime Institute of Biotechnology). Bands were scanned using AlphaView SA version 3.3.0.0 software (ProteinSimple, San Jose, CA, USA). Rabbit polyclonal Beclin 1 antibody (1:1,000; cat. no. 11306-1-AP) was purchased from ProteinTech Group, Inc. (Chicago, IL, USA). Rabbit polyclonal SQSTM/p62 (1:1,000; cat. no. 5114), rabbit polyclonal LC3A/B (1:1,000; cat. no. 4108), rabbit monoclonal phospho-AMPK $\alpha\left(\mathrm{Thr}^{172}\right)$ (1:1,000; cat. no. 2535), rabbit polyclonal AMPK $\alpha(1: 1,000$; cat.no. 2532), rabbit monoclonal phosphor-p70 $0^{\mathrm{s} 6}$ kinase $\left(\mathrm{Thr}^{389}\right)$ (1:1,000; cat. no. 9234) and rabbit polyclonal P70S6 kinase (P70S6K) (1:1,000; cat. no. 9202) antibodies were purchased from Cell Signaling Technology, Inc. (Danvers, MA, USA). Rabbit polyclonal GAPDH antibody (1:7,000; cat. no. ABS16) was purchased from EMD Millipore.

Quantitative polymerase chain reaction ( $q P C R)$. The animal groupings and treatments in each group were as described above. Samples were isolated from the LV muscle of Sham-operated heart (Sham-operation group), the infarct LV muscle of control hearts (I/R group) and the infarct LV muscle of IPostC hearts (IPostC group). Total RNA was isolated using the RNAiso Plus kit (Takara Biotechnology Co., Ltd., Dalian, China) and cDNA was synthesized with $1 \mu \mathrm{g}$ total RNA using gDNA Eraser (Takara Biotechnology Co., Ltd.). A total of $2 \mu \mathrm{l}$ cDNA template was mixed with $18 \mu 12 \mathrm{X} \mathrm{SYBR}{ }^{\circledR}$ Premix EX Taq II mix (Takara Biotechnology Co., Ltd.) and this mixture was subsequently subjected to qPCR at $95^{\circ} \mathrm{C}$ for $30 \mathrm{sec}$, followed by 40 cycles of $95^{\circ} \mathrm{C}$ for $5 \mathrm{sec}$ and $60^{\circ} \mathrm{C}$ for $34 \mathrm{sec}$ in the ABI 7500 Fast Real-Time PCR system (Applied Biosystems Life Technologies, Foster City, CA, USA). GAPDH was amplified as an internal reference gene. The following primers were used for amplification: i) Rat microtubule associated protein 1 light chain 3 (LC3) b (LC3) forward, 5'-AGCTCTGAAGGCAACAGCAACA-3' and reverse, 5'-GCTCCATGCAGGTAGCAGGAA-3'; ii) rat SQSTM1 (p62) forward, 5'-TGTGGTGGGAACTCGCTATAA GT-3' and reverse, 5'-AAAGGGTTGGGAAAGATGAG-3'; iii) rat Beclin 1 forward, 5'-GAAACTGGACACGAGCTT CAAGA-3' and reverse, 5'-ACCATCCTGGCGAG-3'; iv) rat GAPDH forward, 5'-GGCACAGTCAAGGCTGAGAATG-3' and reverse, 5'-ATGGTGGTGAAGACGCCAGTA-3' (Takara Biotechnology Co., Ltd.). The comparative $2^{-\Delta \mathrm{Ct}}$ method (12) was utilized to analyze the relative expression of genes.

Determination of myocardial infarct size. Rats were randomly divided into the I/R, IPostC and IPostC + chloroquine (CQ) groups. Rats in the I/R group were subjected to $30 \mathrm{~min}$ ischemia to the heart followed by 3 -h reperfusion. Rats in the IPostC group were subjected to $30 \mathrm{~min}$ ischemia to the heart prior to three cycles of 30 -sec reperfusion followed by 30 -sec ischemia, and finally $3 \mathrm{~h}$ of reperfusion. Rats in the IPostC + CQ group were pretreated with CQ (10 mg/kg, intraperitoneally) $1 \mathrm{~h}$ prior to the induction of ischemia (13), and were subsequently subjected to the same protocol as rats in the IPostC group ( $n=6-8$ rats per group). Rats were sacrificed by overdose of anesthesia (pentobarbital, $180 \mathrm{mg} / \mathrm{kg}$ ).

To measure the ischemic area at risk (AAR), the LAD was re-occluded with the same suture. Evans blue dye (3\%; Sigma-Aldrich) was injected through the caudal vein. The heart was excised and the LV tissues were cut into 1-mm cross-sections (Leica CM1950 cryostat; Leica Microsystems $\mathrm{GmbH}$, Nussloch, Germany). The LV sections were incubated with a $1 \%$ triphenyltetrazolium chloride solution (Sigma-Aldrich) at $37^{\circ} \mathrm{C}$ for $15 \mathrm{~min}$. The infarct area (pale), the AAR (not blue) and the total LV area from both sides of each section were measured using Image-Pro Plus version 6.0 (Media Cybernetics, Inc., Rockville, MD, USA).

Terminal deoxynucleotidyl transferase mediated dUTP nick end labeling (TUNEL) staining. The hearts were rapidly excised following 3-h reperfusion and were fixed in $4 \%$ paraformaldehyde (Sinopharm Chemical Reagent Co., Ltd., 
A

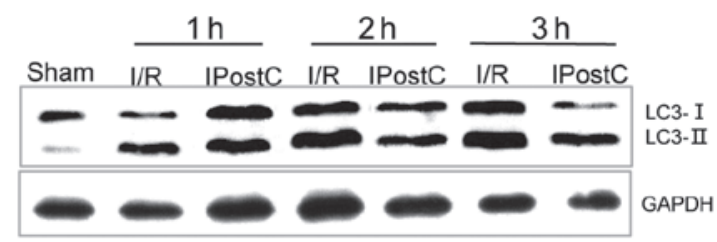

B

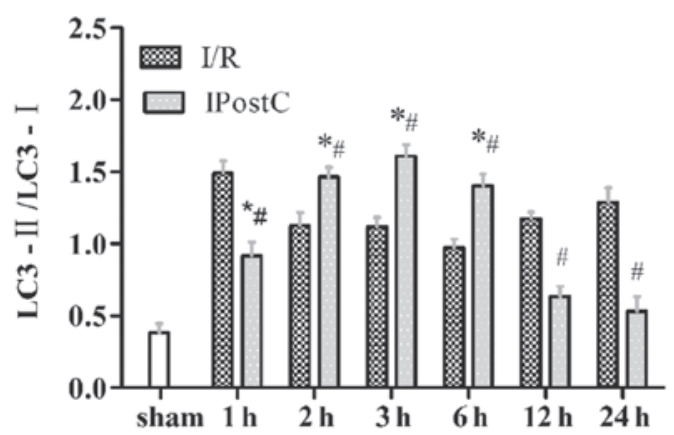

D

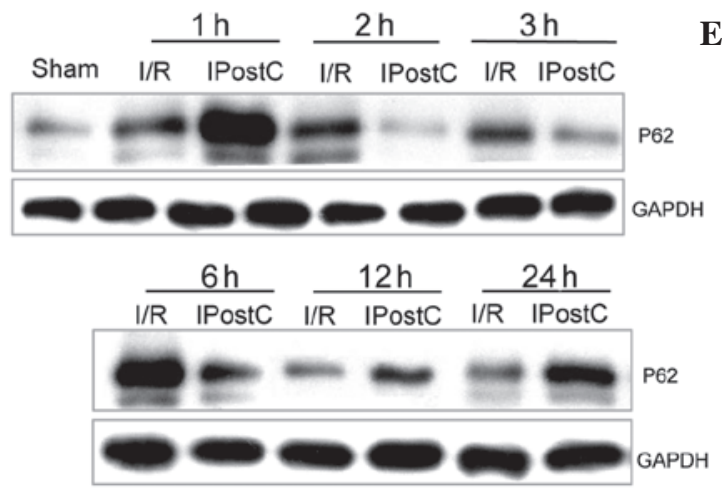

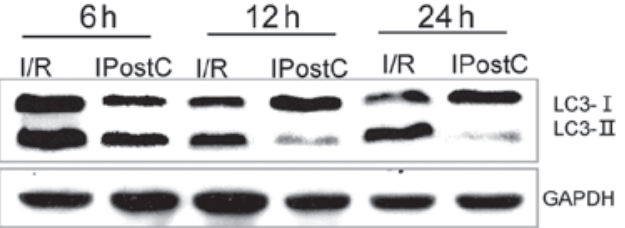

C

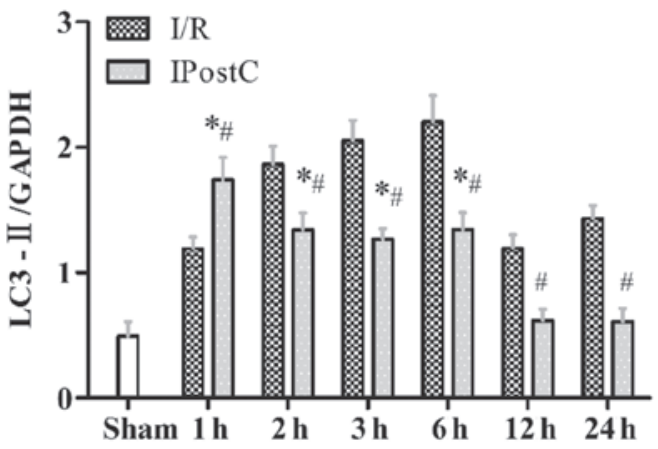

Figure 1. IPostC regulates autophagic activity. Sprague Dawley rats were subjected to $30 \mathrm{~min}$ ischemia followed by reperfusion for the indicated length of time, with or without IPostC treatment. (A) Western blot analysis of LC3 protein expression in ventricular tissue. Quantitative analyses of the ratio of (B) LC3-II/LC3-I and (C) LC3-II/GAPDH. (D) Western blot analysis of p62 protein expression in ventricular tissue. (E) Quantitative analysis of the ratio of p62/GAPDH. Error bars represent the mean \pm standard error of the mean; $n=6$ for each time-point; ${ }^{*} \mathrm{P}<0.05$ vs. Sham group, ${ }^{\text {"P }}<0.05$ vs. I/R group. IPostC, ischemic postconditioning; I/R, ischemia/reperfusion; LC3, microtubule associated protein 1 light chain 3.

Shanghai, China). The $10-\mu \mathrm{m}$ transverse cryostat sections were cut from routinely Tissue-Tek OCT (Sakura Finetek, Inc., Torrance, CA, USA)-embedded heart tissues. The slices were processed for TUNEL staining using a commercially available in situ cell detection kit (Roche Diagnostics, Indianapolis, IN, USA). Nuclei were stained with DAPI (Beyotime Institute of Biotechnology). Apoptotic cell counts were expressed as a percentage of the total number of nuclei.

Statistical analysis. Data were presented as the mean \pm standard error of the mean and were analyzed using SPSS version 17.0 (SPSS, Inc., Chicago, IL, USA). One-way analysis of variance was used for a comparison between multiple groups and the Bonferroni post hoc test was used for pairwise comparisons between groups. $\mathrm{P}<0.05$ was considered to indicate a statistically significant difference.

\section{Results}

IPostC altered autophagic activity and Beclin 1, AMPK-mTOR expression in a time-dependent manner. In the myocardium of rats treated with IPostC compared with those of rats in the I/R group, the LC3-II/LC3-I ratios were significantly lower at 1,12 and $24 \mathrm{~h}$ but significantly greater at 2,3 and $6 \mathrm{~h}(\mathrm{P}<0.05$; Fig. 1A and B). The LC3-II protein levels were significantly upregulated at $1 \mathrm{~h}$ but significantly downregulated at the other time points $(\mathrm{P}<0.05$; Fig. $1 \mathrm{C})$. The p62 protein levels were significantly greater at 1,12 and $24 \mathrm{~h}$ but significantly reduced at 2, 3 and $6 \mathrm{~h}(\mathrm{P}<0.05$; Fig. $1 \mathrm{D}$ and E) Furthermore, the LC3b mRNA levels were observed to be significantly lower at 1 and $24 \mathrm{~h}$ but significantly higher at 3 and $6 \mathrm{~h}$ ( $\mathrm{P}<0.05$; Fig. 2A), and the p62 mRNA levels were also significantly lower at 1 and $24 \mathrm{~h}$ but significantly higher at 2, 3 and $6 \mathrm{~h}(\mathrm{P}<0.05$; Fig $2 \mathrm{~B})$. Overall, IPostC was observed to inhibit autophagic activity within the first hour, promote autophagic activity from 2 to $6 \mathrm{~h}$ and reduce autophagic activity from 12-24 h.

In the IPostC-treated myocardium, the Beclin 1 mRNA levels were significantly lower at $1 \mathrm{~h}$ and significantly higher at $3,6,12$ and $24 \mathrm{~h}$, as compared with the I/R group $(\mathrm{P}<0.05$; Fig. 2C). The expression levels of P70S6K were significantly increased following $1 \mathrm{~h}$, reduced at 2, 3 and $6 \mathrm{~h}$ and increased again at 12 and $24 \mathrm{~h}(\mathrm{P}<0.05$; Fig. $3 \mathrm{~A}$ and $\mathrm{B})$. AMPK activity 
A

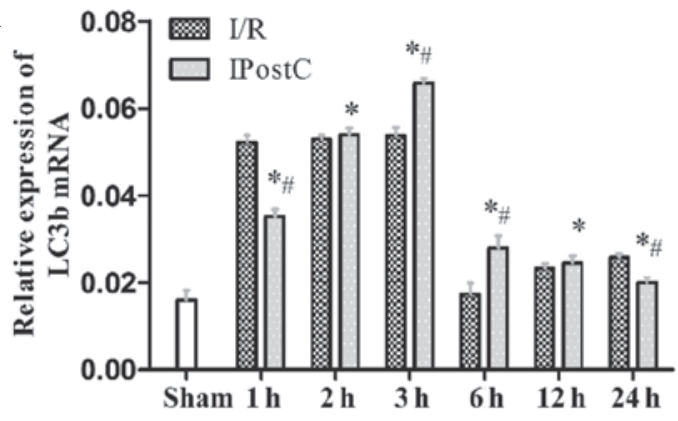

B

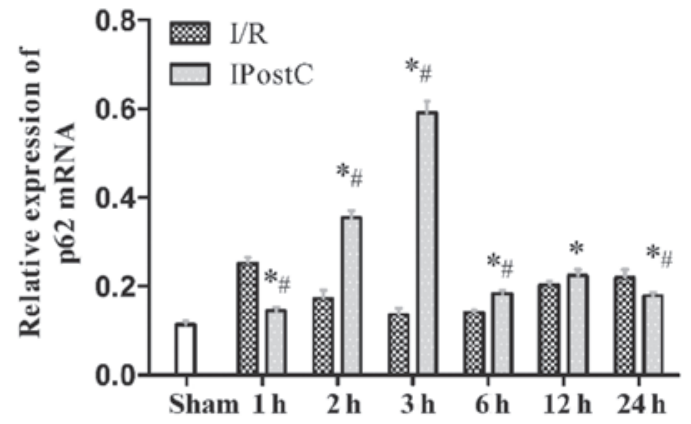

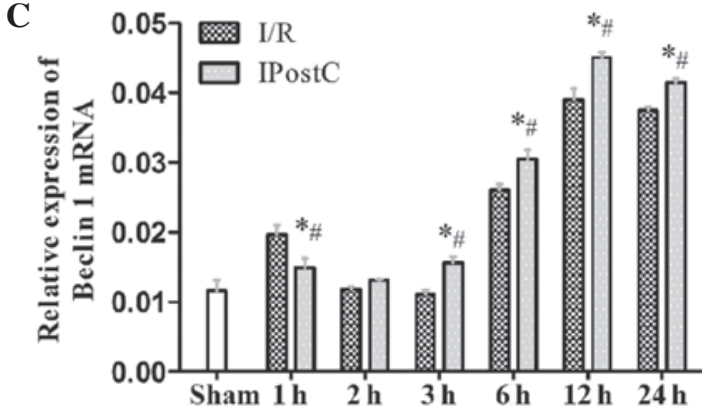

Figure 2. IPostC regulates LC3b, p62 and Beclin 1 gene expression. Alterations in (A) LC3b, (B) p62 and (C) Beclin 1 mRNA expression levels in ventricular tissue. Error bars represent the mean \pm standard error of the mean, $n=6$ rats at each time-point. Data were compared by one-way analysis of variance with the post hoc Bonferroni test. ${ }^{*} \mathrm{P}<0.05$ vs. Sham group, ${ }^{\#} \mathrm{P}<0.05$ vs. I/R group. IPostC, ischemic postconditioning; I/R, ischemia/reperfusion; mRNA messenger RNA.

was significantly inhibited at $1 \mathrm{~h}$, increased at 2,3 and $6 \mathrm{~h}$ and reduced at 12 and $24 \mathrm{~h}(\mathrm{P}<0.05$; Fig. $3 \mathrm{C}$ and $\mathrm{D})$. Beclin 1 protein levels were significantly increased at $1 \mathrm{~h}$, reduced at 2,3 and $6 \mathrm{~h}$, followed by an increase again at 12 and $24 \mathrm{~h}$ $(\mathrm{P}<0.05$; Fig. 3E and F).

Promotion of autophagic flux is significant in conferring IPostC-mediated cardioprotection. A previous study demonstrated that the reperfused myocardium exhibited impaired autophagosome clearance, which is associated with cardiomyocyte death (14). The current study also observed impaired autophagic flux in the reperfused myocardium following 2-6 $\mathrm{h}$ of reperfusion. Whether the relief of autophagy blocked by IPostC in this period serves a significant role in its myocardial protection was also investigated. The anti-malaria drug CQ is a well-known autophagy inhibitor, which inhibits lysosomal acidification and prevents autophagosome-lysosomal fusion (15). Rat hearts were pretreated with CQ $1 \mathrm{~h}$ prior to coronary artery occlusion. Following 3 -h reperfusion, the ischemic AAR was measured. The infarct volume in the hearts subjected to I/R was $52 \%$ of the total LV area and this was reduced to $26 \%(\mathrm{P}<0.05)$ in hearts subjected to IPostC. By contrast, the infarct volume in the IPostC + CQ rats was significantly increased to $53 \%$ of the total $\mathrm{LV}$ area $(\mathrm{P}<0.05$ vs. IPostC group; Fig. 4A and B). Furthermore, the percentage of TUNEL-positive cells in the ischemic area was additionally increased in the CQ-treated myocardium (17\%) compared with that of the IPostC-treated myocardium (7\%) (Fig. 4C and D).

\section{Discussion}

Autophagy has been demonstrated to be upregulated during myocardial I/R and has been suggested to be cardioprotective during ischemia. IPostC has been previously observed to function as a protective mechanism against reperfusion injury (8). However, the role of autophagy in the protective effects of IPostC and the associated signaling pathways remain to be fully elucidated. The results of the current study suggested that IPostC regulates autophagy in a time-dependent manner through the regulation of Beclin 1 and AMPK-mTOR signaling pathways.

Autophagy is a dynamic process and its activity is predominantly reflected by autophagic flux, which is comprised of autophagosome formation and autolysosome clearance (13). The conversion of LC3-I to LC3-II is a marker of autophagic vesicle formation, however high ratios of LC3-II/LC3-I may reflect an increase in autophagy, a reduction in autophagic flux due to impaired fusion with lysosomes or autolysosomal degradation. p62 is a protein adaptor, which is able to bind ubiquitinated cargo designated for autophagic breakdown. LC3-II, which is located on the inner autophagosomal membrane, is degraded when the autophagosome fuses with the lysosome (16). Detecting the LC3-II/LC3-I ratio and levels of p62 and LC3-II protein provides an improved representation of autophagic flux $(6,15)$. p62 and LC3 are able to be transcriptionally regulated during autophagy, which may influence the efficacy of p62 and LC3 levels as indicators of autophagic substrates $(15,17)$. Despite these potential limitations, p62 and LC3b gene expression levels were selected for detection in the myocardium during the reperfusion period by qPCR. The RISK pathway predominantly includes the phosphoinositide 3-kinase/protein kinase B (Akt)/mTOR pathway and p42/p44 extracellular signal-regulated kinase pathway. These two pathways converge at P70S6K. In mammalian cells, phosphorylation of mTOR inhibits cell autophagy (17). Numerous studies demonstrate that mTOR is a key factor for the regulation of autophagy in I/R (18-22). P70S6K is an effector of mTOR, and phosphorylated (p-)P70S6K levels are 
A

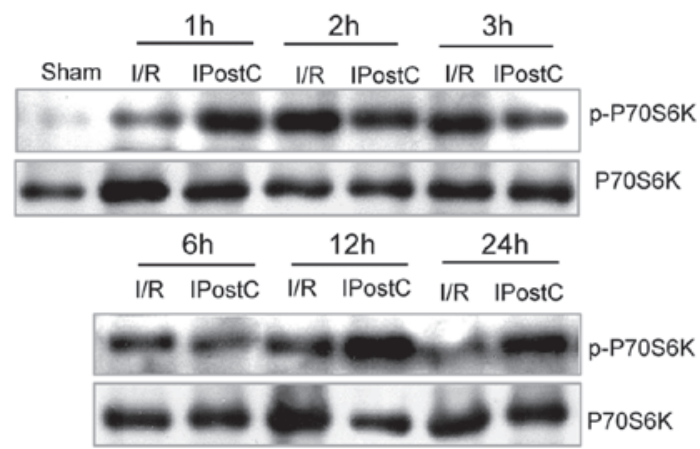

C

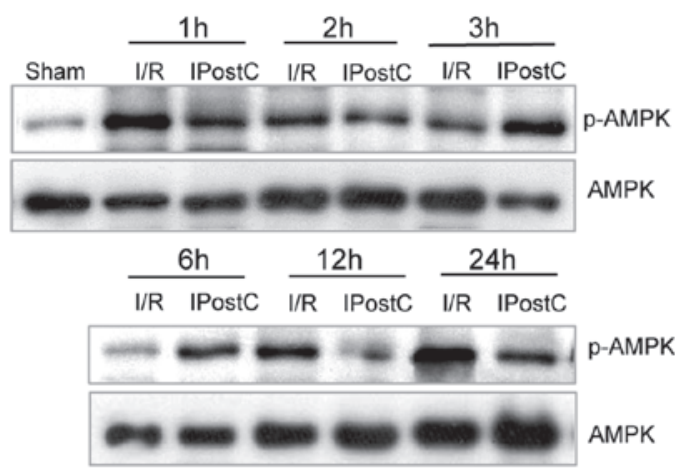

E

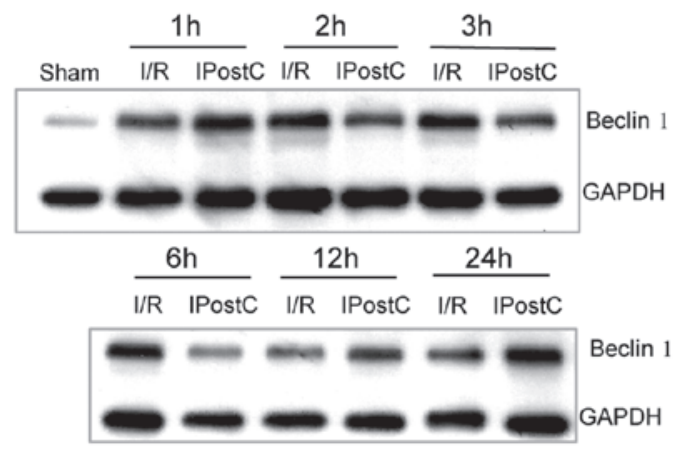

B

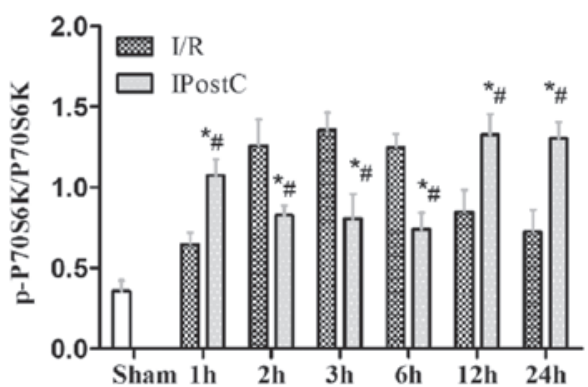

D

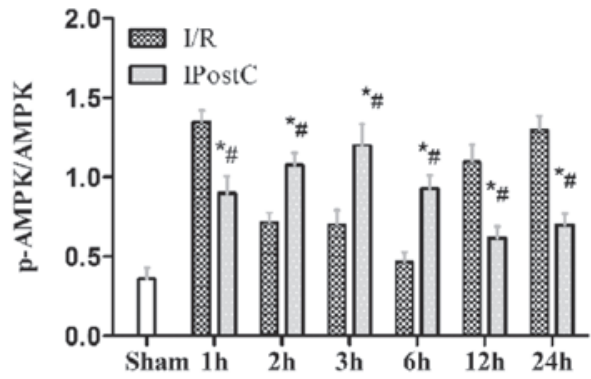

F

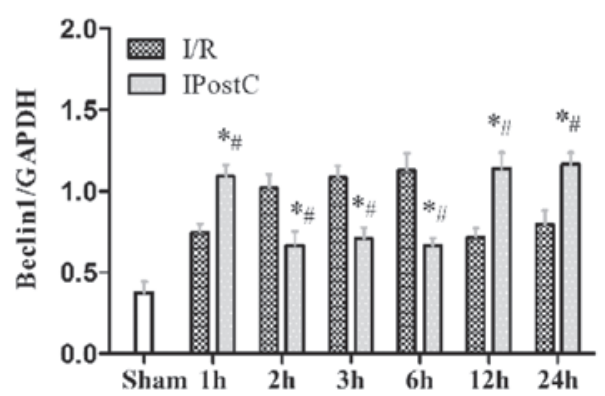

Figure 3. IPostC regulates the Beclin 1 and AMPK-mTOR pathways. Rats were subjected to 30 min ischemia followed by reperfusion for the indicated length of time, with or without IPostC treatment. (A) Western blot analysis of p-P70S6K (Thr ${ }^{389}$ ) protein expression. (B) Quantitative analysis of the ratio of p-P70S6K/P70S6K. (C) Western blot analysis of p-AMPK (Thr ${ }^{172}$ ) protein expression. (D) Quantitative analysis of the ratio of p-AMPK/AMPK. (E) Western blot analysis of Beclin 1 protein expression. $(\mathrm{F})$ Quantitative analysis the ratio of Beclin 1/GAPDH. Error bars represent the mean \pm standard error of the mean; $\mathrm{n}=6$ for each time-point. Data were compared with a one-way analysis of variance and post hoc Bonferroni test; ${ }^{*} \mathrm{P}<0.05$ vs. Sham group, ${ }^{\#} \mathrm{P}<0.05$ vs. I/R group. IPostC, ischemic postconditioning; AMPK, adenosine monophosphate-activated kinase; p-, phosphorylated; P70S6K, P70S6 kinase; I/R, ischemia/reperfusion; mTOR, mammalian target of rapamycin.

suggested to reflect the function of mTOR (23). AMPK is able to directly trigger autophagy or inhibit the suppressive effect of mTOR (24). Beclin 1 is a protein that controls the initiation of autophagy; however, greater Beclin 1 levels additionally impede lysosomal fusion by interacting with Rubicon or by directly downregulating transcriptional components of the autophagy-lysosome machinery $(14,25)$. Therefore, Beclin 1 is a key regulator, which exerts dual-directional regulation on autophagic flux. For these reasons, the markers selected in the current study are appropriate for the identification of autophagy and the associated signaling.

During the first hour following treatment, reduced gene expression of LC3b and a lower LC3-II/LC3-I ratio were observed in the IPostC myocardium, which suggested a reduction in autophagosome formation. Meanwhile, high p62 protein levels with reduced p62 gene expression indicated that IPostC also inhibited autophagy clearance. Therefore, it was suggested that IPostC suppressed autophagic activity within the first hour following its application. Consistent with a previous study (26), P70S6K was observed to be significantly activated within $1 \mathrm{~h}$ following IPostC treatment, suggesting that mTOR was also activated. In addition, a significant reduction in AMPK activity was observed in the IPostC myocardium. Thus, IPostC may negatively regulate autophagy via the activation of mTOR. The expression of the Beclin 1 gene was reduced by IPostC in this first hour, but the Beclin 1 protein level was upregulated in the IPostC myocardium compared with the I/R group myocardium. Upregulation of Beclin 1 has been previously identified to be mediated by the high reactive oxygen species (ROS) content resulting 
A
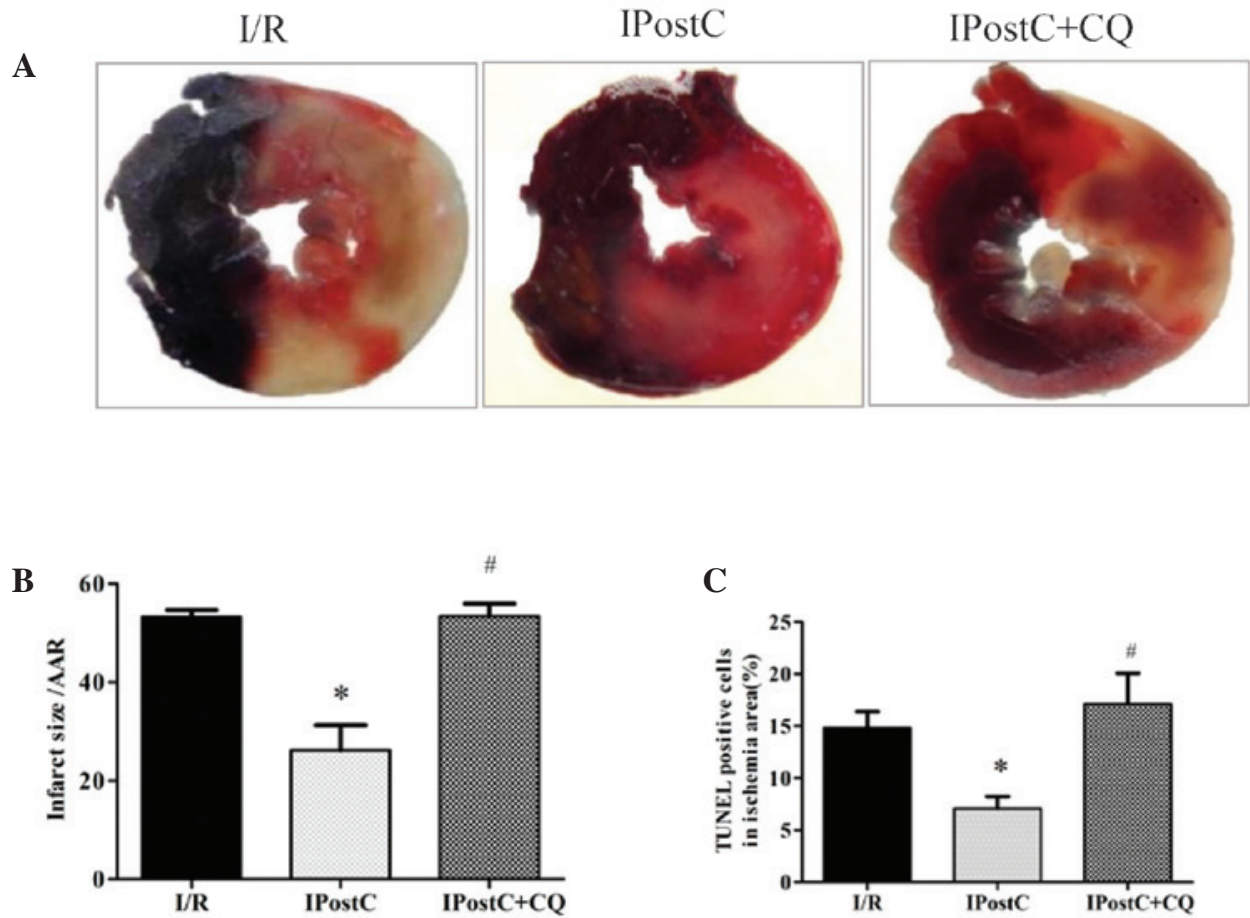

D

TUNEL

DAPI

Merged
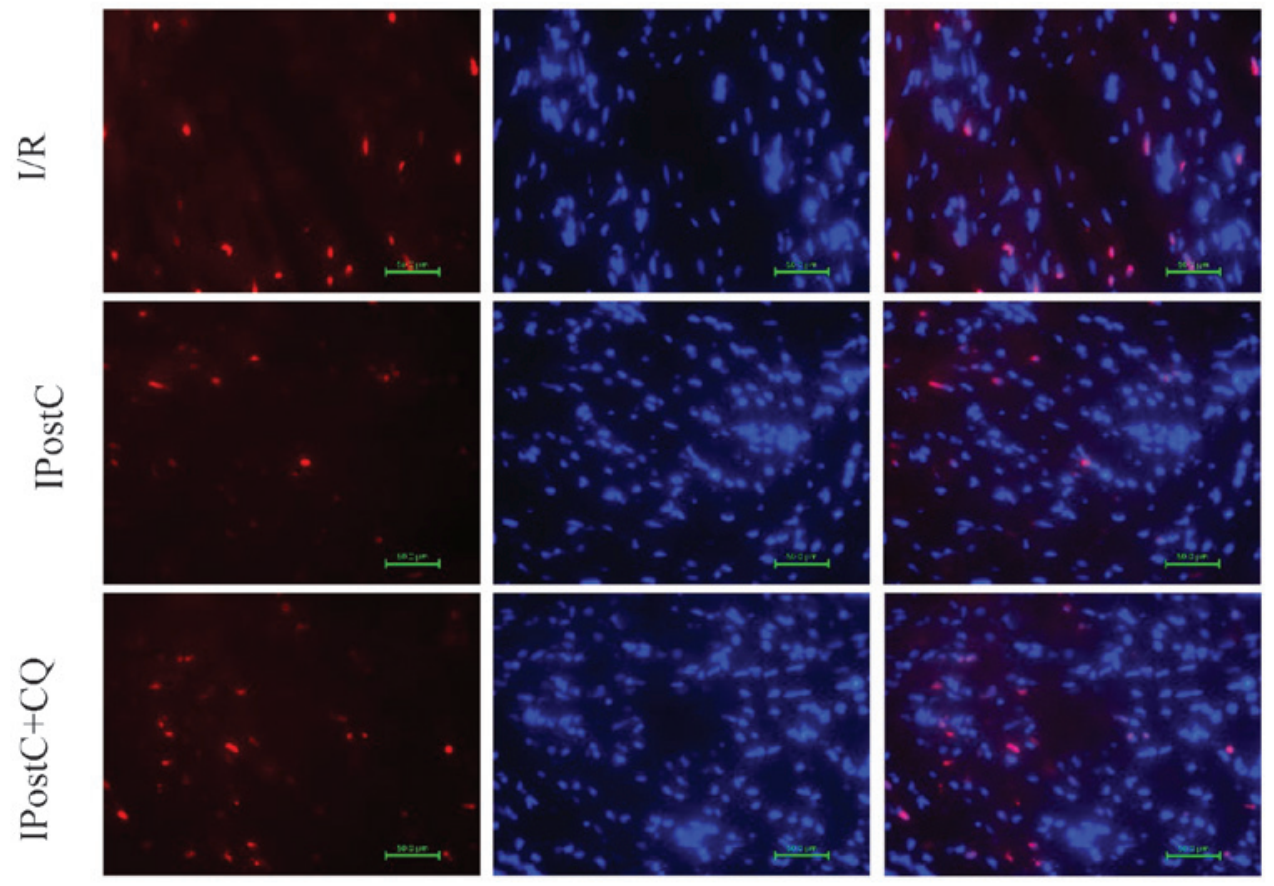

Figure 4. Inhibition of autophagy reduces IPostC cardioprotection. (A) Typical photographs of left ventricle tissue stained by Evans blue and triphenyltetrazolium chloride dye. Rats were subjected to $30 \mathrm{~min}$ of ischemia and $3 \mathrm{~h}$ of reperfusion with or without IPostC and/or CQ treatment. Magnification, $\mathrm{x}$. (B) Quantitative analysis of infarct volume, $\mathrm{n}=6-8$ per group. (C) TUNEL-positive myocytes in the ischemic area. The number of TUNEL-positive myocytes was expressed as a percentage of total nuclei detected by DAPI staining, $n=6$ per group. (D) LV tissue sections were subjected to TUNEL (red) and DAPI (blue) staining, scale bar $=50 \mu \mathrm{m}$. Error bars represent the mean \pm standard error of the mean; data were compared with a one-way analysis of variance and post hoc Bonferroni test; " $\mathrm{P}<0.05$ vs. I/R group, ${ }^{\text {}} \mathrm{P}<0.05$ vs. IPostC group. IPostC, ischemic postconditioning; TUNEL, terminal deoxynucleotidyl transferase mediated dUTP nick end labeling; $\mathrm{I} / \mathrm{R}$, ischemia/reperfusion; $\mathrm{CQ}$, chloroquine; AAR, area at risk.

from reperfusion $(14,27)$. It is known that IPostC is able to reduce the production of $\operatorname{ROS}(26)$, therefore a reduction in the levels of ROS was hypothesized to contribute to the reduced Beclin 1 expression during the first hour following IPostC treatment. A previous study observed that Akt may enhance the interaction among Beclin 1, 14-3-3 and vimentin, which inhibits the role of Beclin 1 in autophagy (28). Therefore, activation of the RISK pathway by IPostC may be caused by 
the accumulation of Beclin 1 in the cytoplasm. The higher concentration of Beclin 1 may then inhibit autophagic activity by impeding autophagy clearance or downregulating the transcription of autophagy-associated genes (14). Autophagy in the I/R myocardium at $1 \mathrm{~h}$ appears to be over-activated when compared with the myocardium in the IPostC group.

The excessive activation of autophagy may have detrimental effects due to a disproportionate degeneration of various key organelles and proteins (29). Thus, it is suggested that the temporary inhibition of autophagy induced by IPostC produced a protective effect on the myocardium.

The LC3-II/LC3-I ratio was observed to be higher in the IPostC myocardium during 2-6 h post-ischemia and the gene expression of LC3b was increased at 2 and $6 \mathrm{~h}$, which suggested that autophagosome formation was increased. In addition, reduced levels of p62 protein expression together with significantly increased p62 gene expression levels indicates that IPostC also promotes autophagy clearance. The LC3-II levels were reduced in the IPostC-treated myocardium, which suggested that autophagy clearance was promoted by IPostC, rather than the blockage of autolysosome clearance induced by reperfusion. During this phase, P70S6K activation was reduced and AMPK was activated again, an effect which appeared to mediate autophagy activation in this period of time. The Beclin 1 protein level was reduced and Beclin 1 gene expression was increased in the IPostC myocardium compared with the reperfused myocardium. The reduced Beclin 1 protein level may have enhanced the autophagic flux during this time. Previous studies have demonstrated that autophagic flux was blocked during reperfusion $(9,14)$. In the current study, it was reported that reperfusion impaired autophagic flux between 2 and $6 \mathrm{~h}$ in I/R, and IPostC enhanced autophagic activity during this period. It was further demonstrated that inhibition of autophagic flux by CQ significantly increased myocardial infarct volume and apoptosis, suggesting that relief of the impaired autophagic flux by reperfusion is important in IPostC-induced cardioprotection. During this period of time the reperfused myocardium exhibited impaired autophagic flux, which may be an endogenous adaptation to the overactivation of autophagy during the first hour; therefore, blocking the autophagic flux temporarily produced a protective effect on the myocardium. However, if this condition persists, it is damaging to the myocardium due to the fact that the heart must remove damaged proteins and organelles, particularly during the reperfusion phase (30). A previous study indicated that impaired autophagy was responsible for cardiomyocyte death (14). The enhanced autophagy induced by IPostC during this period produced a protective effect on the myocardium.

At the 12 and $24 \mathrm{~h}$ time-points, the reduced LC3-II/LC3-I ratio, $\mathrm{LC} 3 \mathrm{~b}$ and p62 gene expression levels together with increased p62 protein expression levels suggested that autophagic activity was reduced in the IPostC myocardium. Simultaneously, P70S6K was observed to be activated once again. Thus, mTOR may be involved in negative regulation of autophagy in the IPostC myocardium. The Beclin 1 protein and gene expression levels were all upregulated in the IPostC myocardium. In addition to participating in autophagy, Beclin 1 is also involved in various physiological processes, functioning as an important membrane binding protein $(31,32)$. Considering the reduced autophagic activity, Beclin 1 may participate in additional physiological processes in the IPostC myocardium at these time-points. Autophagic activity was restored in the reperfused myocardium during the 12-24 h period, potentially as a result of the lower Beclin 1 levels improving autophagic flux or due to the fact that myocardial cells with autophagic dysfunction had already died. The autophagic activity was reduced in the IPostC myocardium due to the fact that the dysfunctional or damaged cellular components had been eliminated and mTOR was re-activated, thus promoting the production of proteins to repair the reperfused myocardium.

In conclusion, the current study highlighted the crucial role of autophagy in the protective mechanism of IPostC via regulation of the Beclin 1 and AMPK-mTOR signaling pathways. However, further studies are required to confirm an association between these signaling pathways and the regulation of autophagy. Regulating autophagy in a time-dependent manner may be an additional important end-effector of IPostC protection. Inhibition or activation of autophagy during reperfusion may be harmful to the myocardium, however regulation of autophagic activity in a time-dependent manner may be an effective novel cardioprotective treatment strategy.

\section{References}

1. Zhao ZQ and Vinten-Johansen J: Postconditioning: reduction of reperfusion-induced injury. Cardiovasc Res 70: 200-211, 2006.

2. Hausenloy DJ: Signalling pathways in ischaemic postconditioning. Thromb Haemost 101: 626-634, 2009.

3. Ovize M, Baxter GF, Di Lisa F, et al; Working Group of Cellular Biology of Heart of European Society of Cardiology: Postconditioning and protection from reperfusion injury: where do we stand? Position paper from the Working Group of Cellular Biology of the Heart of the European Society of Cardiology. Cardiovasc Res 87: 406-423, 2010.

4. Dosenko VE, Nagibin VS, Tumanovskaya LV, Moibenko AA and Vaage J: Postconditioning prevents apoptotic necrotic and autophagic cardiomyocyte cell death in culture. Fiziol Zh 51: 12-17, 2005.

5. Wei C, Li H, Han L, Zhang L and Yang X: Activation of autophagy in ischemic postconditioning contributes to cardioprotective effects against ischemia/reperfusion injury in rat hearts. J Cardiovasc Pharmacol 61: 416-422, 2013.

6. Klionsky DJ, Abdalla FC, Abeliovich H, et al: Guidelines for the use and interpretation of assays for monitoring autophagy. Autophagy 8: 445-544, 2012.

7. Matsui Y, Kyoi S, Takagi H, et al: Molecular mechanisms and physiological significance of autophagy during myocardial ischemia and reperfusion. Autophagy 4: 409-415, 2008.

8. Matsui Y, Takagi H, Qu X, et al: Distinct roles of autophagy in the heart during ischemia and reperfusion: roles of AMP-activated protein kinase and Beclin 1 in mediating autophagy. Circ Res 100: 914-922, 2007.

9. Hamacher-Brady A, Brady NR and Gottlieb RA: Enhancing macroautophagy protects against ischemia/reperfusion injury in cardiac myocytes. J Biol Chem 281: 29776-29787, 2006.

10. Dosenko VE, Nagibin VS, Tumanovska LV and Moibenko AA: Protective effect of autophagy in anoxia-reoxygenation of isolated cardiomyocyte? Autophagy 2: 305-306, 2006.

11. Valentim L, Laurence KM, Townsend PA, et al: Urocortin inhibits Beclin1-mediated autophagic cell death in cardiac myocytes exposed to ischaemia/reperfusion injury. J Mol Cell Cardiol 40: 846-852, 2006

12. Schmittgen TD and Livak KJ: Analyzing real-time PCR data by the comparative C(T) method. Nat Protoc 3: 1101-1108, 2008

13. Iwai-Kanai $\mathrm{E}$, Yuan $\mathrm{H}$, Huang $\mathrm{C}$, et al: A method to measure cardiac autophagic flux in vivo. Autophagy 4: 322-329, 2008.

14. Ma X, Liu H, Foyil SR, et al: Impaired autophagosome clearance contributes to cardiomyocyte death in ischemia/reperfusion injury. Circulation 125: 3170-3181, 2012.

15. Mizushima N, Yoshimori $\mathrm{T}$ and Levine B: Methods in mammalian autophagy research. Cell 140: 313-326, 2010. 
16. Klionsky DJ, Abdalla FC, Abeliovich H, et al: Guidelines for the use and interpretation of assays for monitoring autophagy. Autophagy 8: 445-544, 2012

17. He C and Klionsky DJ: Regulation mechanisms and signaling pathways of autophagy. Annu Rev Genet 43: 67-93, 2009.

18. Pattingre S, Espert L, Biard-Piechaczyk M and Codogno P: Regulation of macroautophagy by mTOR and Beclin 1 complexes. Biochimie 90: 313-323, 2008.

19. Kim J, Kundu M, Viollet B and Guan KL: AMPK and mTOR regulate autophagy through direct phosphorylation of Ulk1. Nat Cell Biol 13: 132-141, 2011.

20. Jung CH, Ro SH, Cao J, Otto NM and Kim DH: mTOR regulation of autophagy. FEBS Lett 584: 1287-1295, 2010.

21. Yu L, McPhee CK, Zheng L, et al: Termination of autophagy and reformation of lysosomes regulated by mTOR. Nature 465: 942-946, 2010.

22. Alers S, Löffler AS, Wesselborg S and Srork B: Role of AMPK-mTOR-Ulk1/2 in the regulation of autophagy: Cross talk, shortcuts, and feedbacks. Mol Cell Biol 32: 2-11, 2012.

23. Tsang CK, Qi H, Liu LF and Zheng XF: Targeting mammalian target of rapamycin (mTOR) for health and diseases. Drug Discov Today 12: 112-124, 2007.

24. Mihaylova MM and Shaw RJ: The AMPK signalling pathway coordinates cell growth, autophagy and metabolism. Nat Cell Biol 13: 1016-1023, 2011.
25. Zhong Y, Wang QJ, Li X, et al: Distinct regulation of autophagic activity by Atg14 L and Rubicon associated with Beclin 1-phosphatidylinositol-3-kinase complex. Nat Cell Biol 11: 468-476, 2009.

26. Penna C, Mancardi D, Rastaldo R and Pagliaro P: Cardioprotection: a radical view Free radicals in pre and postconditioning. Biochim Biophys Acta 1787: 781-793, 2009.

27. Hariharan N, Zhai P and Sadoshima J: Oxidative stress stimulates autophagic flux during ischemia/reperfusion. Antioxid Redox Signal 14: 2179-2190, 2011.

28. Wang RC, Wei Y, An Z, et al: Akt-mediated regulation of autophagy and tumorigenesis through Beclin 1 phosphorylation. Science 338: 956-959, 2012

29. Gustafsson $\AA \mathrm{B}$ and Gottlieb RA: Autophagy in ischemic heart disease. Circ Res 104: 150-158, 2009.

30. Gottlieb RA, Finley KD and Mentzer RM Jr: Cardioprotection requires taking out the trash. Basic Res Cardiol 104: 169-180, 2009.

31. Huang W, Choi W, Hu W, et al: Crystal structure and biochemical analyses reveal Beclin 1 as a novel membrane binding protein Cell Res 22: 473-489, 2012.

32. Funderburk SF, Wang QJ and Yue Z: The Beclin 1-VPS34 complex-at the crossroads of autophagy and beyond. Trends Cell Biol 20: 355-362, 2010. 